\title{
È cambiato qualcosa nelle indicazioni all'ablazione con radioiodio del residuo tiroideo nel carcinoma differenziato della tiroide?
}

\author{
Raffaella Forleo $^{1}$ - Maria Grazia Castagna ${ }^{1}$
}

Accettato: 30 luglio 2020 / Pubblicato online: 29 gennaio 2021

(c) The Author(s) 2021

\section{Introduzione}

La terapia con 131-I (RAI), quando effettuata nei pazienti con carcinoma differenziato della tiroide (CDT) sottoposti a tiroidectomia totale (TxT) a scopo ablativo o adiuvante, ha come finalità l'eradicazione di eventuali residui tiroidei e/o microfocolai di carcinoma presenti nel tessuto tiroideo. In aggiunta consente, attraverso l'effettuazione della scintigrafia totale corporea, la precoce individuazione di persistenza di malattia locoregionale e/o a distanza.

Ad oggi, nella pratica clinica, le indicazioni per la terapia ablativa sono diventate più selettive e sono basate sul rischio di persistenza/recidiva di malattia del paziente definito in accordo con la classificazione TNM VII ed. e proposto dalle linee guida dell'American Thyroid Association (ATA) nel 2015 [1]. Nel 2018 sei diverse società scientifiche italiane hanno pubblicato una Consensus [2] sulla gestione e il trattamento dei pazienti con CDT nella quale le indicazioni al RAI sono basate sul TNM VIII ed. [3], pubblicato nel 2016 ed entrato nella pratica clinica nel 2018, che ha introdotto importanti novità rispetto alla VII ed. del 2009. Di particolare interesse è la differente definizione della categoria T3 (Tabella 1) che comprende nella VIII ed. tumori di diametro massimo (Dmax) $>4 \mathrm{~cm}$ (T3a) e tumori con grossolana estensione extratiroidea ai muscoli striati del collo (T3b) senza più menzione alla minima estensione extratiroidea (MEE) ai tessuti lassi peritiroidei [3]; di conseguenza, i tumori con MEE vengono classificati solo in accordo al loro Dmax e pertanto gran parte dei tumori, precedentemente classificati come T3, vengono ora ricollocati nelle cate-

M.G. Castagna

mariagrazia.castagna@unisi.it

1 Dipartimenti di Scienze Mediche, Chirurgiche e Neuroscienze, UOC di Endocrinologia, Policlinico Santa Maria alle Scotte, Università di Siena, Siena, Italia gorie $\mathrm{T} 1 \mathrm{o} \mathrm{T} 2$, così come già dimostrato da molti studi in letteratura $[4,5]$.

\section{Definizione del rischio di persistenza/recidiva e indicazioni alla terapia ablativa con 131-I secondo la Consensus italiana}

L'impiego della VIII ed. del TNM nella Consensus italiana ha introdotto alcune importanti modifiche nella definizione del rischio di recidiva/persistenza di malattia rispetto al sistema proposto dall'ATA. Nella classe "rischio basso", per cui non vi è indicazione al RAI, sono inclusi i pazienti con tumore tiroideo di Dmax $\leq 2 \mathrm{~cm}$, senza metastasi locali e/o a distanza (T1a-b, N0-X, M0-X). Nella categoria "rischio basso-intermedio o intermedio" sono inclusi pazienti senza metastasi a distanza, con tumore di Dmax $\leq 2 \mathrm{~cm}$ con metastasi linfonodali o con tumore $>2 \mathrm{~cm} \mathrm{e} \leq 4 \mathrm{~cm}$ con o senza metastasi locali (T1-2, N1a-b, M0-X). In questo gruppo di pazienti è suggerito un uso selettivo della terapia con 131I: la decisione è pertanto lasciata al clinico e deve essere basata sulle caratteristiche clinico-patologiche (età avanzata, tumore esteso, linfonodi macroscopici o clinicamente evidenti, estensione extranodale, istologia aggressiva o invasione vascolare) associate a un outcome sfavorevole. Sono considerati a rischio alto i pazienti con macroscopica estensione extra-tiroidea e/o con metastasi a distanza (T3-4, qualsiasi $\mathrm{N}$, qualsiasi $\mathrm{M}$; qualsiasi $\mathrm{T}$, qualsiasi $\mathrm{N}, \mathrm{M} 1$ ) e pertanto il RAI è sempre raccomandato [2].

Come indicato nella Tabella 2, una prima differenza che emerge tra il sistema di stratificazione del rischio della Consensus italiana rispetto all' ATA è l'inclusione dei tumori T2, con o senza metastasi locali, nella classe a rischio intermedio e non più nella classe di rischio bassa. Inoltre, i tumori 
Tabella 1 Sistema di stadiazione AJCC/TNM - VII-VIII edizione

\begin{tabular}{|c|c|c|c|}
\hline \multicolumn{2}{|c|}{ TNM VII edizione } & \multicolumn{2}{|c|}{ TNM VIII edizione } \\
\hline T0 & Nessuna evidenza di tumore primario & T0 & Nessuna evidenza del tumore primario \\
\hline T1a & Tumore $\leq 1 \mathrm{~cm}$, senza estensione extratiroidea & T1a & Tumore $\leq 1 \mathrm{~cm}$ nel diametro massimo, limitato alla tiroide \\
\hline $\mathrm{T} 1 \mathrm{~b}$ & $\begin{array}{l}\text { Tumore }>1 \mathrm{~cm} \text { ma con } \leq 2 \mathrm{~cm} \text { nel diametro massimo, senza } \\
\text { estensione extratiroidea }\end{array}$ & $\mathrm{T} 1 \mathrm{~b}$ & $\begin{array}{l}\text { Tumore }>1 \mathrm{~cm} \mathrm{ma} \leq 2 \mathrm{~cm} \text { nel diametro massimo, limitato } \\
\text { alla tiroide }\end{array}$ \\
\hline $\mathrm{T} 2$ & $\begin{array}{l}\text { Tumore }>2 \mathrm{~cm} \mathrm{ma} \leq 4 \mathrm{~cm} \text { nel diametro massimo, senza } \\
\text { estensione extratiroidea }\end{array}$ & $\mathrm{T} 2$ & $\begin{array}{l}\text { Tumore }>2 \mathrm{~cm} \mathrm{ma} \leq 4 \mathrm{~cm} \text { nel diametro massimo, limitato } \\
\text { alla tiroide }\end{array}$ \\
\hline \multirow[t]{2}{*}{$\mathrm{T} 3$} & \multirow{2}{*}{$\begin{array}{l}\text { Tumore }>4 \mathrm{~cm} \text { nel diametro massimo limitato alla tiroide o } \\
\text { qualsiasi dimensione del tumore con minima estensione } \\
\text { extratiroidea (es. estensione al muscolo sternotiroideo o ai } \\
\text { tessuti lassi peritiroidei) }\end{array}$} & $\mathrm{T} 3 \mathrm{a}$ & Tumore $>4 \mathrm{~cm}$ nel diametro massimo limitato alla tiroide \\
\hline & & $\mathrm{T} 3 \mathrm{~b}$ & $\begin{array}{l}\text { Tumore di qualsiasi dimensione con macroscopica estensione } \\
\text { extra-tiroidea, invasione dei muscoli striati (sternotiroideo, } \\
\text { sternoioideo o omoioideo) }\end{array}$ \\
\hline $\mathrm{T} 4 \mathrm{a}$ & $\begin{array}{l}\text { Tumore di qualsiasi dimensione esteso oltre la capsula } \\
\text { tiroidea con invasione dei tessuti lassi sottocutanei, laringe, } \\
\text { trachea, esofago o nervo laringeo ricorrente }\end{array}$ & $\mathrm{T} 4 \mathrm{a}$ & $\begin{array}{l}\text { Tumore esteso oltre alla capsula tiroidea e che invade tutti i } \\
\text { tessuti lassi sottocutanei, laringe, trachea, esofago, nervo } \\
\text { laringeo ricorrente. }\end{array}$ \\
\hline $\mathrm{T} 4 \mathrm{~b}$ & $\begin{array}{l}\text { Tumore di qualsiasi dimensione che invade la fascia } \\
\text { prevertebrale o che ingloba l'arteria carotide o i vasi } \\
\text { mediastinici }\end{array}$ & $\mathrm{T} 4 \mathrm{~b}$ & $\begin{array}{l}\text { Tumore che invade la fascia prevertebrale, i vasi mediastinici } \\
\text { o ingloba l'arteria carotide }\end{array}$ \\
\hline
\end{tabular}

Tabella 2 Comparazione del Sistema di stratificazione del rischio e indicazioni alla terapia ablativa secondo l'ATA e la Consensus Italiana

\begin{tabular}{llll}
\hline Classe di rischio & $\begin{array}{l}\text { TNM VII ed. } \\
\text { Linee guida ATA }\end{array}$ & $\begin{array}{l}\text { TNM VIII ed. } \\
\text { Consensus italiana }\end{array}$ & $\begin{array}{l}\text { Indicazione RAI secondo le linee } \\
\text { guida ATA - Consensus Italiana }\end{array}$ \\
\hline BASSA & $\begin{array}{l}\text { T1a-b, N0-X, M0-X } \\
\text { T2, N0-X, M0-X }\end{array}$ & T1a-b, N0-X, M0-X & Non raccomandata di routine \\
BASSO-INTERMEDIA & T3, N0-X, M0-X & T2, N0-X, M0-X & Considerata \\
& T1-3, N1a, M0-X & T1-2, N1a-N1b, M0-X & Indicata di routine \\
\hline
\end{tabular}

con MEE, ora classificati T1 in accordo al diametro tumorale secondo il TNM VIII ed., sono considerati a rischio basso e non più intermedio (ex categoria T3) e, pertanto, non presentano indicazione al RAI. Infine, i tumori nelle categorie $\mathrm{T} 3 \mathrm{a}$ e $\mathrm{T} 3 \mathrm{~b}$ vengono sempre considerati a rischio alto e, pertanto, con indicazione al RAI.

Un recente studio ha validato le indicazioni della Consensus italiana su 380 pazienti con CDT trattati con TxT e RAI [6]. In questo lavoro, l'applicazione della Consensus nella pratica clinica ha portato a una significativa riduzione dei pazienti da sottoporre a terapia radiometabolica postchirurgica con un rischio di ritardata diagnosi di persistenza di malattia minimo e sovrapponibile a quello osservato con l'impiego delle raccomandazioni dell' ATA (1,1 vs 2,1\%, $p=0,37)$. In particolare, la percentuale dei pazienti senza indicazioni alla terapia ablativa passava dal $38,2 \%$ (in accordo con l'ATA) al 47,4\% (in accordo con la Consensus italiana) e quella con raccomandazione al trattamento dall' 1,8 all' $8,2 \%$, rispettivamente. Di conseguenza, la percentuale dei pazienti nei quali veniva consigliato l'uso selettivo del radioiodio diminuiva dal 60 al 44,5\% [6].

\section{Conclusioni}

La Consensus italiana ha modificato in maniera significativa la distribuzione dei pazienti con CDT nelle diverse classi di rischio e, di conseguenza, anche le indicazioni al trattamento ablativo con radioiodio nel post-intervento. La sua applicazione nella pratica clinica riduce significativamente il numero dei pazienti da trattare con RAI dopo la TxT con un minimo rischio di mancata diagnosi di malattia metastatica.

Funding Note Open Access funding provided by Università degli Studi di Siena within the CRUI-CARE Agreement.

Conflitto di interesse Le autrici Raffaella Forleo e Maria Grazia Castagna dichiarano di non avere conflitti di interesse.

Consenso informato Lo studio presentato in questo articolo non ha richiesto sperimentazione umana.

Studi sugli animali Le autrici di questo articolo non hanno eseguito studi sugli animali.

Nota della casa editrice Springer Nature rimane neutrale in riguardo alle rivendicazioni giurisdizionali nelle mappe pubblicate e nelle affiliazioni istituzionali. 
Open Access This article is licensed under a Creative Commons Attribution 4.0 International License, which permits use, sharing, adaptation, distribution and reproduction in any medium or format, as long as you give appropriate credit to the original author(s) and the source, provide a link to the Creative Commons licence, and indicate if changes were made. The images or other third party material in this article are included in the article's Creative Commons licence, unless indicated otherwise in a credit line to the material. If material is not included in the article's Creative Commons licence and your intended use is not permitted by statutory regulation or exceeds the permitted use, you will need to obtain permission directly from the copyright holder. To view a copy of this licence, visit http://creativecommons.org/licenses/by/4.0/.

\section{Bibliografia}

1. Haugen BR, Alexander EK, Bible KC et al (2016) 2015 American Thyroid Association management guidelines for adult patients with thyroid nodules and differentiated thyroid cancer: the American Thyroid Association guidelines task force on thyroid nodules and differentiated thyroid cancer. Thyroid 26(1):1-133
2. Pacini F, Basolo F, Bellantone R et al (2018) Italian consensus on diagnosis and treatment of differentiated thyroid cancer: joint statements of six Italian societies. J Endocrinol Invest 41(7):849-876

3. Amin MB, Edge SB, Greene FL et al (2017) AJCC cancer staging manual, 8th edn. Springer, New York

4. Kim M, Kim WG, Oh HS et al (2017) Comparison of the seventh and eighth editions of the American Joint Committee on Cancer/Union for International Cancer Control tumor-nodemetastasis staging system for differentiated thyroid cancer. Thyroid 27(9):1149-1155

5. Tuttle RM, Haugen B, Perrier ND (2017) Updated American Joint Committee on Cancer/Tumor-Node-Metastasis staging system for differentiated and anaplastic thyroid cancer (eighth edition): what changed and why? Thyroid 27(6):751-756

6. Forleo R, Fralassi N, Maino F et al (2020) Indication for radioiodine remnant ablation in differentiated thyroid cancer patients: does 2018 Italian consensus change anything? J Endocrinol Invest 41(7):849-876 